\title{
HIGH RESOLUTION FLEXIBLE TACTILE SENSORS
}

\author{
DRIMUS, A[lin] M[arian] \& BILBERG, A[rne]
}

\begin{abstract}
This paper describes the development of a tactile sensor for robotics inspired by the human sense of touch. It consists of two parts: a static tactile array sensor based on piezoresistive rubber and a dynamic sensor based on piezoelectric PVDF film. The combination of these two layers addresses both spatial distribution of pressure and dynamic events such as contact, release of contact and slip. Data acquisition and object recognition applications are described and it is proposed that such a sensor could be used in robotic grippers to improve object recognition, manipulation of objects and grasping.
\end{abstract}

Key words: tactile sensors, piezoresistive materials, robotics, $P V D F$, piezoelectricity, object recognition

\section{INTRODUCTION}

Unstructured environments require handling of potentially unknown objects and in such cases vision is not always able to provide all the information needed in order to manipulate these objects. Complementary to vision, humans use their sense of touch to perceive mechanical properties of objects, such as texture, elasticity, compliance or mass. Force and tactile sensing at the finger-object contact are essential for fine manipulation and complex tasks with changing grasp requirements (Howe, 94).

When it comes to building tactile sensors for robots, it comes naturally to seek inspiration from biology, because humans perform extraordinary in many tasks that require extensive use of the sense of touch. Mechanoreceptors are the units that convert tactile stimuli into neuron excitations and are of two types: static - Ruffini corpuscles and Merkel receptors and dynamic - Meissner cells and Pacini corpuscles. Therefore tactile interaction can be described by two complementary modalities: the dynamic sensing, where we deal with responses caused by changes in the condition of contacts, based on movements of the fingers, vibrations or slip occurance and static sensing, where the distribution of tactile mechanoreceptors is used to determine local surface shape and pressure distribution. There are a few technologies that can be used for manufacturing tactile sensors and the most used are piezoresistive (rubbers or inks), piezocapacitive, piezoelectrical and optical (Cutkosky et al., 2008). Our work considers the static tactile arrays based on piezoresistive technology and the dynamic sensors based on piezoelectric materials.

Compared to vision, there has not been a huge progress into the manufacturing of tactile sensors and we are still far from an 'artificial skin' tactile sensor. The biggest problems concern the difficulty of wiring and fragility of such sensors, not to mention the cost and difficulty of customization (Cutkosky et al., 2008). The requirements for a tactile sensor array similar to an artificial skin would be:

- conformability and thin, to suit any kind of finger or gripper

- spatial sensing resolution and sensitivity similar to the human skin

- robustness and repeatability for industrial use

In the last decades, quite a few sensor prototypes have been developed. Flexible sensors based on pressure conductive rubber with $3 \times 16$ cells were developed using a stitched electrode structure, but the construction method and the leak currents bring high variations in the measurements (Shimojo et al., 2004). Industrial tactile sensors have been developed by Weiss (Weiß \& Wörn, 2005) but they are not flexible and have low resolution, $6 \times 14$ cells in $2.4 \mathrm{~cm} \times 5 \mathrm{~cm}$. A flexible $16 \times 16$ sensor array with $1 \mathrm{~mm}$ spatial resolution was developed for minimal invasive surgery, but the sensor fails to give steady output for static stimuli, and has a high hysteresis and nonlinearity (Goethals et al., 2008). A combination of static and dynamic sensor was developed to address both pressure profiles and slippage, but the design has only $4 \times 7$ cells, and a number of wires equal to the number of cells (Göger et al., 2009).

\section{DEVELOPMENT OF TACTILE SENSOR}

\subsection{Piezoresistive rubber}

The piezoresistive rubber is a thin sheet of a rubberlike material that changes its electrical resistance according to the induced strain caused by application of mechanical load. It consists of an elastomer that has electric conductive particles dispersed and by applying a pressure, the distance between the particles decreases and because of the percolation theory it will result in more ways for the current to flow, thus decreasing the electrical resistance. The behaviour of one single cell based on this material is showed in Figure 1 for 20 trials of increasing and decreasing the force. It can be seen that a nonlinear effect and hysteresis are present, but this is similar to the response in the human sense of touch.

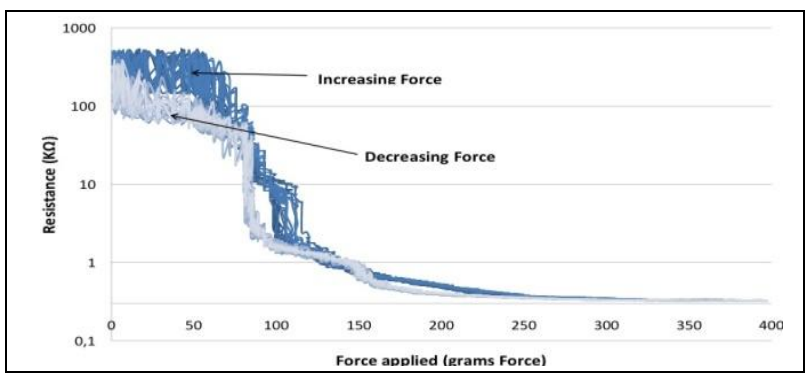

Fig. 1. Response of a tactile cell during 20 trials of applying increasing and decreasing $0-400$ grams force.

\subsection{Building a tactile array}

To address arrays of cells with minimum wiring complexity we consider the use of a rows and columns approach. This technique implies equally spaced rows of electrodes on the face of the material, followed by a perpendicular arrangement of equally spaced columns on the back of the material. This sandwich structure decreases the number of wires to a minimum compared to a normal array, from $2 \times n^{2}$ to $2 \times n$ wires. In our case for a tactile array of 512 cells only 48 wires are necessary $(32 \times 16)$. The tactile array sensor prototype is 6 $\mathrm{cm} \times 3 \mathrm{~cm}$, where each cell has a size of $1.6 \mathrm{~mm} \times 1.6 \mathrm{~mm}$ and one taxel is close to the size of the mechanoreceptors in the human skin. Figure 2 represents the schematic for a $4 \times 4$ array. This approach stars with a flexible polymer (PVC) substrate as 
the base layer covered with an adhesive layer. A conductive paint (flexible polymer with silver particles) is applied in a thin layer through a mask with patterns for electrodes. After the mask is removed, the paint bounds to the base substrate. After the paint cures, we add the piezoresistive patch on top of the base layer with electrodes and we complete the "sandwich" like structure with another layer similar to the base layer, with the electrodes facing inwards and such that the top electrodes and the bottom electrodes are perpendicular. The layout is depicted in Figure 2. The limitations of this prototype are at the interfacing level (fragile interconnects) and due to the high sensitivity threshold.

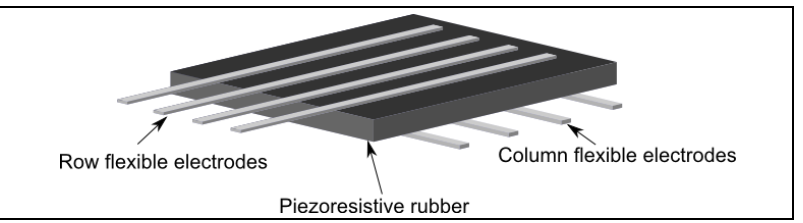

Fig. 2. Layout of a $4 \times 4$ array sensor for static sensing

\subsection{Piezoelectric PVDF film}

PVDF (Polyvinylidene fluoride) film is a flexible and thin kind of macromolecule piezo materials. The response is quite similar to the signal variation of the Pacinian corpuscle sensory receptor in the dermis and the output is characterized by the temporal differential property. The output follows a brief potential wave when a pulse of pressure is applied and a similar pulse when pressure is released. However, there is no response if the pressure stimuli are stationary. The structure of the film is presented in Figure 4.a) and this dynamic layer is positioned below the static layer in the final sensor prototype.

\subsection{Data acquisition}

Data acquisition is realized using a dsPIC33 that applies a voltage over one of the 32 rows and reads all outputs from the 16 columns. Using a multiplexing algorithm, all rows and columns are addressed at a rate providing 100 tactile images per second. The output from the dynamic sensor is amplified using a charge amplifier and output voltages are read at a rate of 600 samples per second.

\section{APPLICATIONS OF THE SENSOR}

\subsection{Object recognition}

Object recognition can be performed using the haptic data, when grasping small objects. In our case the images in Figure 3 show objects (like a screw, a washer, and two connectors that were pressed against the tactile sensor with even pressure) and the corresponding tactile images produced as the output (pressure maps). Due to the high resolution of the sensor, different small objects can be recognized using computer vision algorithms applied on haptic data.

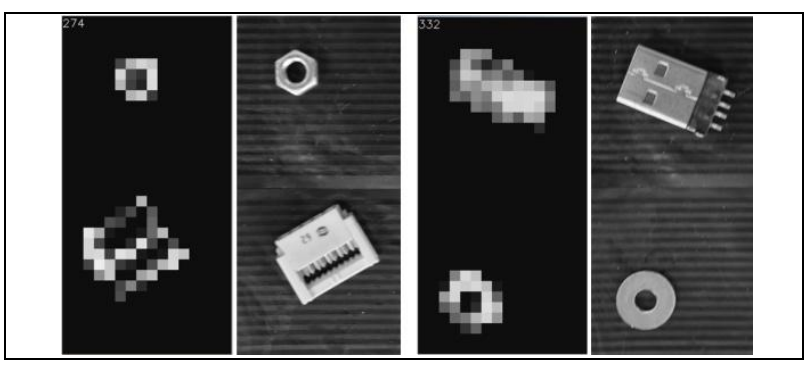

Fig. 3. Tactile images of small objects placed on the sensor

\subsection{Event detection}

Due to the relatively high sensitivity threshold of the static sensor it is not be possible to detect gentle contact (as the touch of a needle). However, this can be detected using the dynamic sensor, which gives a high output for gentle contact. Another important state is the release of contact, which can be recognized with its negative peak. When active sensing is performed (rubbing the robotic finger across a textured object) vibrations occur and by an FFT analysis of the time signal, the texture of the object can be extracted. It is also possible to detect the incipient slip due to vibrations perceived and prevent slip. The output signal is depicted in Figure 4.b).

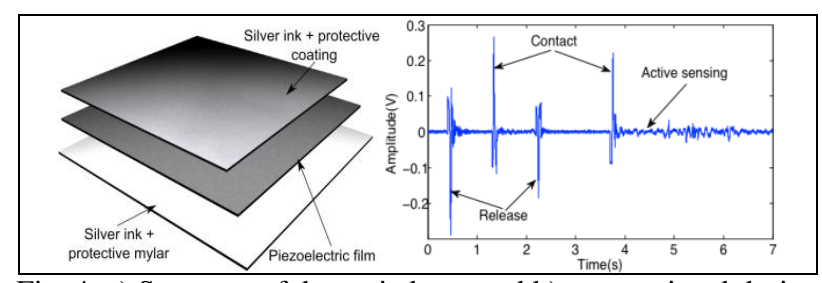

Fig. 4. a) Structure of dynamic layer and b) output signal during different events: contact, release and sliding the surface against a rough object

\section{CONCLUSION}

In this article, we have presented the development of a tactile sensor inspired by the two modalities of the human sense of touch. The sensor consists of two layers, one for static stimuli and one for dynamic stimuli. The static layer is built using a thin and flexible piezoresistive rubber and the dynamic layer is based on piezoelectric PVDF films. Data acquisition is realized using fast multiplexing techniques for the static layer and a charge amplifier for the dynamic layer. We exemplify applications of the two sensing modalities in terms of object recognition based on contact shape and event detection in robotic manipulation. Future work will investigate how to increase the sensitivity in the static layer, improve the manufacturing method, dynamic signal analysis for slip detection and perform object recognition based on haptic data.

\section{ACKNOWLEDGEMENTS}

This project is part of the Handyman Project, a Danish initiative supported by the Danish National Advanced Technology Foundation.

\section{REFERENCES}

Cutkosky, M.; Howe, D. \& Provancher, W. (2008) Force and tactile sensors, Springer Handbook of Robotics, pp. 455476

Goethals, P.; Sette, M.; Reynaerts, D. \& Van Brussel, H (2008). Flexible Elastoresistive Tactile Sensor for Minimally Invasive Surgery. Lecture Notes in Computer Science, Springer Berlin 2008, pp 573-579

Göger, D.; Gorges, N. \& Wörn, H. (2009). Tactile Sensing for an Anthropomorphic Robotic Hand: Hardware and Signal Processing, IEEE International Conference on Robotics and Automation

Howe, R.D. (1994). Tactile sensing and control of robotic manipulation, Advanced Robotics, 1994, vol. 8, no. 3, pp 245-261

Schneider, A.; Sturm, J.; Stachniss, C.; Reisert, M. ; Burkhardt, H. \& and Burgard,W. (2009). Object identification with tactile sensors using bag-of-features. In Proc. IEEE International Conference on Intelligent Robots and Systems (IROS), Oct. 2009

Shimojo, M.; Namiki, A.; Ishikawa, M.; Makino, R.\& Mabuchi, K. (2004). A tactile sensor sheet using pressure conductive rubber with electrical wires stitched method, IEEE Sensors Journal, 2004 vol. 4, no. 5, pp. 589-596

Weiß, K. \& Wörn, H. (2005). The Working Principle of Resistive Tactile Sensors Cells, Proc. IEEE International Conference on Mechatronics \& Automation, July 2005 